\title{
L'olivo e l'olivastro de Vincenzo Consolo : pour une odysée du désastre
}

L'olivo e l'olivastro di Vincenzo Consolo: per un'odissea del disastro

Vincenzo Consolo's novel L'olivo e l'olivastro: an odyssey of disaster

\section{Lise Bossi}

\section{OpenEdition}

\section{Journals}

Édition électronique

URL : http://journals.openedition.org/cei/505

DOI : 10.4000/cei.505

ISSN : 2260-779X

\section{Éditeur}

UGA Éditions/Université Grenoble Alpes

Édition imprimée

Date de publication : 15 mars 2012

Pagination : 201-212

ISBN : 978-2-84310-222-6

ISSN : 1770-9571

\section{Référence électronique}

Lise Bossi, «L'olivo e l'olivastro de Vincenzo Consolo : pour une odysée du désastre », Cahiers d'études italiennes [En ligne], 14 | 2012, mis en ligne le 15 septembre 2013, consulté le 27 mars 2021. URL : http://journals.openedition.org/cei/505; DOI : https://doi.org/10.4000/cei.505 


\title{
L'OLIVO E L'OLIVASTRO DE VINCENZO CONSOLO : POUR UNE ODYSÉE DU DÉSASTRE
}

\author{
Lise Bossi \\ Université Paris 4
}

\begin{abstract}
Sulla scena ci sembra sia rimasto solo il coro che in tono alto, poetico, in una lingua non più comunicabile, commenta e lamenta la tragedia senza soluzione, il dolore senza catarsi ${ }^{\mathrm{I}}$.
\end{abstract}

Publié en 1994, L'olivo e l'olivastro de Vincenzo Consolo² reconstitue les étapes d'une nouvelle Odyssée, entendue à la fois comme voyage de retour, comme nostos dans l'espace réel, et comme voyage fantastique dans l'espace de la littérature et de la poésie, pour l'un de " ceux qui sont nés par hasard dans l'île aux trois angles " $(O O$, p. 22). Mais cette Odyssée, largement autobiographique, rêvée initialement comme un retour vers une sicilienne Ithaque d'affection et de mémoire, se transforme bientôt en un voyage dans le désastre qui s'est consommé pendant cette époque atroce qu'a été, pour la Sicile comme pour l'Italie tout entière, la période des années quatre-vingt.

Et nous sommes conviés à suivre le voyageur, à travers « une île perdue, une Ithaque damnée " $(O O, \mathrm{p} .80)$, où tout ce qui subsiste de ce qu'il a connu et aimé est conservé par des érudits et des poètes, qui combattent les prétendants à coup de chantiers de fouilles et de mots écrits noir sur noir, ou par des sortes de gardiens de cimetières verghiens qui ont arrêté le temps en régressant vers une illusoire Troie retrouvée (p. 53).

I. La citation en exergue est extraite de Di qua dal faro, Milan, Mondadori, 1999, p. 262.

2. V. Consolo, L'olivo e l'olivastro, Milan, Mondadori, I994; ci-après, OO. La pagination renvoie à l'édition de poche : V. Consolo, L'olivo e l'olivastro, Oscar Scrittori del Novecento, Milan, Mondadori, I999. 
C'est justement cette Troie, " lieu de pure existence, de simple hasard " $(O O$, p. 49), celle du fallacieux âge d'or à laquelle s'accrochent les Malavoglia, que le narrateur a voulu fuir lorsqu'il a quitté la Sicile à la fin des années soixante pour aller vers ce qu'il appelle les lieux de l'histoire. Vers Palerme, d'abord, « le lieu où se croisent les cultures et les idiomes les plus divers» (p. I23); puis, lorsqu'il a eu le sentiment que toute la Sicile n'était plus qu'un désert historique et social, vers Milan, "dans un contexte urbain dont il ne possédait ni la mémoire, ni le langage » $\left(S I M\right.$, p. $\left.176^{3}\right)$. Et lorsque Milan est devenu l'emblème de « la triste, aliénée et féroce nouvelle Italie du massacre de la mémoire, de l'identité, de la décence et de la civilisation, l'Italie corrompue, barbare, de la mise à sac, des spéculations, de la mafia, des attentats, de la drogue, des voitures, du football, de la télévision et des lotos, du tapage et des poisons » $(O O, \mathrm{p}$. 7I), alors, celui qui écrit a eu le désir de réduire la fracture qui déchirait sa vie en accomplissant une sorte de "voyage pénitentiel » (p. 20) afin de revenir, après plus de vingt ans, au point de départ (p. I2O).

À ceci près qu'il ne s'agit pas, pour «l'éternel Ulysse, le voyageur errant à travers l'île qui fut autrefois son Ithaque » $(O O$, p. I4I), de se laisser réabsorber « par cette nature et cette histoire suspendues, par cette ensorcelante immobilité " (p. I22) qu'il a quittées jadis en se bornant à constater, sur le mode nostalgique et plaintif, qu'il ne retrouve que quelques vestiges de la Sicile qu'il a aimée. Il s'agit d'abord et surtout d'affronter « les ennemis réels, les ennemis historiques qui se sont installés dans sa maison » (p. 20), en dénonçant ce qu'ils ont fait de son Ithaque et, métaphoriquement ${ }^{4}$, de toute l'Italie, au cours des deux dernières décennies, celles des années soixante-dix et quatre-vingt.

Pour cette double tâche d'évocation et de dénonciation, Consolo fait un choix poétique difficile car il prend consciemment le risque mortel « de sortir du récit, de nier la fiction " $(O O, \mathrm{p} .77)$, contrairement à certains de ses compatriotes, tel son ami Sciascia en particulier, qui ont cru pou-

3. V. Consolo, Il sorriso dellignoto marinaio, Turin, Einaudi, 1976 ; ci-après, SIM. Ce texte sera à nouveau publié avec une postface intitulée : "nota dell'autore, ventáani dopo ", Milan, Mondadori, 1997. La pagination renvoie à l'édition de poche : V. Consolo, Il sorriso dell'ignoto marinaio, Oscar Scrittori del Novecento, Milan, Mondadori, 2002. Le texte de la postface que nous citons ici et auquel nous reviendrons plus loin est paru aussi dans Vincenzo Consolo, Di qua dal faro, ouvr. cité ; ci-après, $D Q D F$. La pagination renvoie à l'édition de poche : V. Consolo, Di qua dal faro, Oscar Scrittori del Novecento, Milan, Mondadori, $200 I$.

4. Outre que par Consolo, le fait que la Sicile et la situation sicilienne soient devenues une métaphore de ce qui se passe dans l'Italie tout entière après la seconde guerre mondiale et la trahison des idéaux de la Résistance, est illustré par Sciascia, en particulier dans son ouvrage intitulé la Sicilia come metafora, Milan, Mondadori, 1979, ouvrage qui présente la caractéristique très significative d'avoir été d'abord publié en France sous le titre La Sicile comme métaphore, conversations en italien avec Marcelle Padovani, Paris, Stock, I979. 
voir se servir des instruments de la littérature de masse pour dénoncer les dérives de la société dont la littérature de masse est le produits' Il choisit en outre, comme un autre Ulysse sicilien, comme Verga, d'inventer une langue. Mais, alors que la langue de Verga « a comme imprimé le positif italien sur un négatif lexical et syntaxique dialectal " $(D Q D F$, p. II9), celle de Consolo est prise dans l'épaisseur de toutes les langues de toutes les cultures qui se sont succédées et imbriquées dans l'île, pour mieux en dire et en préserver la réalité, au risque d'être, comme Verga, " détesté à cause de sa langue extrême " $(O O$, p. 58$)$ et de devoir un jour se réfugier dans la solitude, dans l'aphasie, ce qui signifierait, ce qui signifie peut-être déjà, que les monstres ne sont plus des fruits du sommeil ou du remords mais "de vraies menaces, des catastrophes réelles et imminentes» (p. 58).

Ce n'est donc pas la thématique existentielle de l'exil et du retour qui justifie, à elle seule, la référence constamment explicite au voyage initiatique et expiatoire d'Ulysse, c'est aussi que l'Odyssée est d'abord et avant tout un poème et que Consolo entend, dans le droit fil de l'expérimentation littéraire qu'il conduit depuis des années déjà ${ }^{6}$, défendre et illustrer un nouvel épos et un nouveau logos, tissés, comme la toile de Pénélope, avec tous les fils de la mémoire rassemblés pour résister aux usurpateurs et à leurs créatures monstrueuses.

"Ora non può narrare ${ }^{7}$. Tels sont les mots qui ouvrent un texte qui refuse effectivement la linéarité du récit, son développement sur un axe temporel unique et la hiérarchie qui régit les rapports entre le narrateur et ceux qui devraient rester des personnages, entre sa voix dominante et leurs voix secondaires.

À une seule exception près, à laquelle nous reviendrons, celui qui écrit le fait à la troisième personne, en se définissant justement comme «celui qui écrit» $(O O, \mathrm{p} .77)$ ou comme le voyageur. Un voyageur écrivant dont l'existence pourrait relier, et relie parfois, anecdotiquement, les fragments

5. Nous faisons en particulier allusion ici aux quatre grands romans où Sciascia a utilisé, en les subvertissant, les règles et les modalités narratives du genre policier pour dénoncer la subversion de l'État de droit par les représentants de l'État ; romans que l'on peut donc considérer comme les ouvrages fondateurs de ce que l'on appelle aujourd'hui "il noir mediterraneo" ou "noir d'inchiesta" : Il giorno della civetta, Turin, Einaudi, I961 ; A ciascuno il suo, Turin, Einaudi, 1966 ; Il contesto, Turin, Einaudi, I971 ; Todo modo, Turin, Einaudi, 1974 ; auxquels on peut ajouter son tout dernier roman : Una storia semplice, Milan, Adelphi, 1989.

6. Outre que dans la postface à Il sorriso dell'ignoto marinaio précédemment citée, Consolo développe les axes principaux de sa poétique plurilingue et multiculturelle dans un certain nombre des articles du recueil $D i$ qua dal faro; particulièrement dans la section « Sicilia e oltre ", p. 2II-248.

7. Dans cette partie de notre étude, consacrée à l'écriture et à la langue de Consolo, nous avons choisi de conserver le texte original pour certaines citations particulièrement représentatives du rythme et du caractère "mistilingue" de sa prose. 
de ce qui ne peut pas et ne veut pas devenir un récit, pour la bonne raison que son voyage personnel dans l'espace circonscrit de l'île est aussi un voyage à travers d'autres vies et dans d'autres temps, voire dans le non-lieu et le non-temps, dans l'utopie et l'uchronie littéraires.

L'ouvrage est en effet conçu comme une succession de tranches de vie que chacun de ceux qui les ont vécues vient exposer tour à tour. Beaucoup d'entre elles sont issues de la réalité, qu'il s'agisse de la vie ordinaire des émigrants anonymes poussés par les caprices de la nature ou par la misère à quitter une terre ébranlée par les tremblements de terre et ravagée par les éruptions volcaniques ou saignée par la corruption et les exactions mafieuses; ou bien qu'il s'agisse de la vie, dédoublée ou redoublée par leurs œuvres, d'artistes et d'écrivains emblématiques : Antonello da Messina ( $O O$, p. Io) et le Caravage dont les tableaux proposent des paysages d'amour et de mémoire sur lesquels plane déjà l'ombre de la corruption et de la mort (p. 86-97) ; mais aussi Verga, qui a vécu l'exil et le retour « in un'isola che non era l'Itaca dell'infanzia, la Trezza della memoria, ma la Catania pietrosa e inospitale, emblema d'ogni luogo fermo o imbarbarito, che mai lo riconobbe come l'esule che torna, come il figlio » (p. 58) et Sciascia, à qui Vittorini avait prédit qu'il serait emprisonné dans la forme de celui qui reste en Sicile (p. I6). Ou encore Pirandello qui pensait, au début du siècle dernier, que "quel presente burrascoso e incerto [...], ebbro d'eloquio osceno, poteva essere rappresentato solo col sorriso desolato, con l'umorismo straziante, con la parola che incalza e che tortura, la rottura delle forme, della struttura" (p. 67).

Et c'est bien parce que Consolo est convaincu de vivre, lui aussi, un présent tempétueux qu'il rompt à son tour les structures du récit en intercalant entre ces tranches de vie, et en résonance avec elles, des moments de sa propre vie, mais aussi des tranches de vie empruntées à ces œuvres et à ces textes littéraires, sous forme d'évocations presque incantatoires ou de citations ; en entremêlant aux passages de l'Odyssée, origine de toutes les odyssées du monde, des fragments de I Malavoglia, par exemple, parce que même si « la "casa del nespolo" n’a jamais existé [...] les personnages, les personnes, les Malavoglia de toutes les Trezza du monde ont existé » (OO, p. 50).

Tous ces fragments d'être, capturés dans toutes les époques et tous les milieux, habitent et animent chaque lieu visité par le voyageur. Toutes ces voix se mêlent dans une polyphonie où chaque personnage de la nouvelle épopée, du nouvel épos qui nous est proposé, peut se faire entendre et continuer à exister dans une sorte d'éternel présent qui est celui de la mémoire personnelle et littéraire. Et celui qui écrit peut dire qu'il est à la 
fois "l'astuto inventore degli inganni, il guerriero spietato, l'ambiguo indovino, il re privato dell'onore, il folle massacratore degli armenti [...], l'assassino di [...] sua figlia" (OO, p. I) ; il peut dire la peine de Maruzza qui, " madre ammantata, immobile avanti al mare, ai marosi, priva di lacrime, lamento, parola [...], si porta le mani nei capelli, urla nera nel cuore (p. 47); il peut dire qu'il est né à Gibellina et " ha lasciato nelle baracche la madre e la sorella [...]. La sorella più non parla, sì e no con la testa è il massimo che dice " (p. 9-Io). Ainsi, coryphée à la voix plurielle, il redonne une voix à chacun des membres de cette humanité multiple, littéraire ou réelle, pour que les hommes du temps présent les entendent et se souviennent d'eux.

C'est pourquoi il ne veut pas être seulement un nouvel Ulysse qui en racontant "diventa l'aedo e il poema, il cantore e il canto, il narrante e il narrato, l'artefice e il giudice [...], l'inventore di ogni fola, menzogna, l'espositore impudico e coatto d'ogni suo terrore, delitto, rimorso "(OO, p. 19). Car, tel Ulysse avec son bagage de remords et de peine, il a atteint « le point le plus bas de l'impuissance humaine, de la vulnérabilité » et il va devoir choisir entre " la perte de soi, l'anéantissement dans la nature et le salut au sein d'une société, d'une culture»(p. I7-I8), entre l'oléastre et l'olivier. Car, comme l'inventeur du "monstre technologique » (p. 20), qui du meilleur peut faire le pire, de l'instrument de la victoire l'instrument du désastre, du progrès la barbarie, il fait lui aussi partie de cette humanité ambiguë dont Ulysse, le plus humain des héros grecs, est le plus parfait représentant.

C'est justement contre ce désastre et cette barbarie dont il découvre les plaies à chaque étape de son périple autour de l'île que le voyageur Consolo, devenu bâtisseur d'épopée, a voulu dresser le rempart de toutes les vies et de toutes les voix stratifiées qu'il a convoquées dans son Odyssée moderne, un fragment après l'autre, un mot après l'autre. Un rempart à l'image de l'histoire de la Sicile, condamnée par la géographie à subir l'histoire ${ }^{8}$, et qui a connu au cours des siècles une infinité de maux, qu'il s'agisse des tremblements de terre ou des éruptions de l'Etna, des rivalités entre colonies voisines ou des invasions constantes, mais dont les villes détruites par les secousses ou les coulées de lave ont été relevées, à l'instar de Catane dont les habitants sont revenus « a ricostruire mura, rialzare colonne, portali, recuperare torsi, rilievi, mescolando epoche, stili, epigrafi, idoli, in una babele, in una sfida spavalda e irridente " (OO, p. 57). Et Syracuse a su devenir, malgré les invasions ou grâce à elles, "la molteplice città, di cinque nomi, d'antico fasto, di potenza, d'ineguagliabile bellezza, di re sapienti e di tiranni

8. Expression empruntée à L. Sciascia, Cruciverba, Turin, Einaudi, 1983, p. 176. 
ciechi, di lunghe paci e rovinose guerre, di barbarici assalti e di saccheggi: in Siracusa è scritta come in ogni città d'antica gloria, la storia dell'umana civiltà e del suo tramonto" (p. 83-84).

Et c'est justement à Syracuse que celui qui écrit mesure l'abîme qui sépare la ville de ses souvenirs, l'île où, " voyageur solitaire le long d'un itinéraire de connaissance et d'amour, par les sentiers de l'Histoire, il vagabonda pendant un lointain été " ( $O O$, p. I43), et la ville présente, l'île damnée, métaphore de l'Italie fascisante des années quatre-vingt (p. I40). C'est de part et d'autre de l'omphalos d'Ortygie que les deux réalités, la passée et la présente, se distinguent l'une de l'autre, c'est "dans l'espace en forme d'œil, dans la pupille de la nymphe, sur la place où règne la mấtresse de la lumière et de la vue " (p. 83), que, à l'instar du Caravage sur le visage de son page, le coryphée voit, comme dans un miroir déformant, fleurir « la vermeille, la noire tache de la peste, de la corruption et de la fin» (p. 92).

Bien sûr le voyageur pourrait, au risque de se comporter comme " un presbite di mente che guarda al remoto ormai perduto, si ritrae in continuo dal presente, sciogliere un canto di nostalgia d'emigrato a questa città della memoria sua e collettiva, a questa patria d'ognuno chè Siracusa, ognuno che conserva cognizione dell'umano, della civiltà più vera, della cultura "(OO, p. 84). Mais il ne veut pas de ce repli sur un hypothétique âge d'or : "Odia ora. Odia la sua isola terribile, barbarica, la sua terra di massacro" (p. I05). Car désormais, non seulement ce que les caprices de la nature détruisent n'est plus reconstruit mais, de surcroît, la spéculation immobilière et l'industrialisation sauvage achèvent de faire disparaître, en les recouvrant d'une dernière strate mortifère, les témoignages d'une culture millénaire et les beautés d'un patrimoine naturel incomparable. Comme à Augusta «che gli appare nella luce cinerea, nella tristezza di un'Ilio espugnata e distrutta, nella consunzione dell'abbandono, nell'avvelenamento di cielo, mare, suolo " (p. 34). Comme à Milazzo où « sulla piana dove pascolavano gli armenti del Sole, dove si coltivava il gelsomino, è sorta una vasta e fitta città di silos, di tralicci, di ciminiere che perennamente vomitano fiamme e fumo" (p. 28). Et le cancer qui ronge les lieux se propage et corrompt aussi les habitants (p. II7). Comme à Gela où est née non seulement la ville " dell'edilizia selvaggia e abusiva, delle case di mattoni e tondini lebbrosi in mezzo al fango e all'immondizia di quartieri incatastati, di strade innominate, la Gela dal mare grasso d'oli, dai frangiflutti di cemento [ma anche] la Gela della perdita d'ogni memoria e senso, del gelo della mente e dell'afasia" (p. 79). Comme à Avola dont la place géométrique et lumineuse est 
vuota, deserta, sfollata come per epidemia o guerra, rotta nel silenzio dal rombare delle motociclette che l'attraversano nel centro per le sue strade ortogonali, occupata [...] da mucchi di giovani $[. .$.$] che fumano, muti e vacui fissano la vacuità della piazza come in$ attesa di qualcuno, di qualcosa che li salvi. O li uccida. Cosa è successo in questa vasta solare piazza d'Avola? Cosè successo nella piazza di Nicosia, Scicli, Ispica, Modica, Noto, Palazzolo, Ferla, Floridia, Ibla? Cos'è successo in tutte le belle piazze di Sicilia, nelle piazze di quest'Italia d'assenza, ansia, di nuovo metafisiche, invase dalla notte, dalle nebbie, dai lucori elettronici dei video della morte? (OO, p. II2)

Cos'è successo, dio mio, cosè successo a Gela, nell'isola, nel paese in questo atroce tempo? Cosè successo a colui che qui scrive, complice a sua volta o inconsapevole assasssino? Cos’è successo a te che stai leggendo? (OO, p. 8I)

Que s'est-il passé, effectivement, pour que celui qui écrit se prenne lui-même à partie dans une sorte de dédoublement où sa voix semble se dissocier de sa plume et lance ce "Dio mio » qui n'est pas une exclamation vide de sens mais un véritable cri de douleur; un cri de douleur à travers lequel Consolo, car c'est bien de lui qu'il s'agit ici, trahit, pour la seule et unique fois tout au long de cette Odyssée polyphonique, l'engagement qu'il s'est fixé de n'être que le porte-voix et le porte-plume, de ne jamais dire, contrairement à Pausanias, "Io sono il messaggero, l'anghelos, sono il vostro medium, a me è affidato il dovere del racconto: conosco i nessi, la sintassi, le ambiguità, le malizie della prosa, del linguaggio" (OO, p. 39). Pausanias qui représente, dans le texte de Consolo, les Proci, les prétendants de la naissante littérature postmoderne qui se font les complices, à moins qu'ils n'en soient les fauteurs, de l'assassinat de la culture et de la mémoire par le pouvoir politico-médiatique déjà tout-puissant depuis un certain décret de 1983. Pausanias à qui Empédocle, dont Consolo reprend à son compte l'approche sensorielle et poétique de la connaissance et la philosophie du savoir révélé par le logos, rétorque :

Che menzogna, che recita, che insopportabile linguaggio! È proprio il degno figlio di quest'orrendo tempo, di questo abominevole contesto, di questo gran teatro compromesso, di quest'era soddisfatta, di questa società compatta, priva di tradimento, d'eresia, priva di poesia. Figlio di questo mondo degli avvisi, del messaggio tondo, dei segni fitti del vuoto. (OO, p. 40)

Que s'est-il passé pour que, après avoir engagé directement sa responsabilité en tant qu'écrivant, celui qui écrit apostrophe ainsi le lecteur et l'accuse d'être le complice de l'assassinat du logos par les Proci de la littérature de masse?

Peut-être la réponse se trouve-t-elle dans ce qu'ils ont tous en commun : le langage, l'écriture, les mots en somme?

Peut-être tout cela a-t-il commencé dans les mots, par les mots? 
C'est en tout cas ce que Consolo entend démontrer, comme Sciascia l'avait fait, en 1978, à l'occasion de sa magistrale enquête philologique sur les documents relatifs à l'Affaire Moro'. Une enquête où il donnait raison à Pasolini qui, dans son célèbre article de 1975, dit "l'article des lucioles", affirmait déjà que " comme toujours, ce n'est que dans la langue que sont apparus les premiers symptômes ». "Les symptômes, commente Sciascia, de la course vers le vide de ce pouvoir démocrate-chrétien qui avait été, jusqu'à dix ans auparavant, la continuation pure et simple du régime fasciste. " $(A M$, p. I5)

Les mêmes symptômes s'étaient justement manifestés dans les années qui avaient précédé la montée du fascisme, et la transformation du langage en gesticulation oratoire, en rhétorique patriotarde ${ }^{10}$, avait déjà été un signe avant-coureur, une préfiguration de la corruption du corps social et de la vie publique par la peste fasciste. Alors, la langue que Verga avait forgée pour son poème narratif, son " épopée populaire " $(O O, \mathrm{p} .48)$, s'était abîmée dans "la retorica sicilianista, l'equivoco, l'alibi regressivo e dialettale dei mafiosi, dei baroni e dei poetastri " (p. 77), ou s'était perdue dans l'aphasie et le silence, devant "l'eloquio vano, prezioso e abbagliante di D’Annunzio, [...] i giochi spacconi e insensati dei futuristi " (p. 59).

De la même façon, les malheurs de Gela ont commencé lorsque, au lieu d'encourager, après l'unanimisme fasciste, ce qui aurait pu être un nouveau Risorgimento culturel et linguistique, la plupart des intellectuels italiens, pour des raisons largement idéologiques, ont préféré, comme Visconti, tourner des films tels que La terra trema, où " la lingua inventata da Verga regrediva in dialetto, in suono incomprensivo, in murmure di fondo " $(O O$, p. 50) ou bien considérer, comme Vittorini, que la découverte de pétrole dans les tombes grecques et les citernes sarrasines de ce petit village de pêcheurs et la naissance de Gela I, Gela 2, Gela 3 et de la Gulf Italia Company méritaient d'être célébrées à grand renfort de « volenterosa poesia, retorica industriale, lombarda e progressiva» (p. 78).

Le résultat de ces choix esthétiques et politiques, dont Consolo n'exclut pas, comme on l'a vu, que lui-même et le lecteur aient pu être les complices, s'affichent sur le visage de la Gela des années quatre-vingt et la misère culturelle et morale dans laquelle vivent ses habitants se reflète dans le spectacle de désolation qu'elle offre au voyageur. La misère des plus jeunes, en particulier, qui n'ont eu pour seuls repères que ceux qui leur

9. L. Sciascia, L'affaire Moro, Palermo, Sellerio, I978; ci-après $A M$.

Io. Sciascia avait analysé les raisons politiques et sociologiques de ce qu'il considérait, déjà, comme une dérive irréversible de la langue et de la littérature vers la confusion et le vide dans I9I2+I, Milan, Adelphi, I986, p. I3-I6. 
ont été fournis par «la furbastra e volgare letteratura sulla degradazione e la marginalità sociale, sul male di Gela, di Licata, di Palma di Montecchiaro, di Canicatti o di Palermo servito in serials televisivi, in Piovra I, Piovra 2, Piovra 3 [e nei] libri di vuote chiacchiere, di stanca ecolalia sui mali di Sicilia" $(O O$, p. 80$)$. Quant à ceux qui n'ont même plus ces repères-là, il ne leur reste que "il linguaggio turpe della siringa e del coltello, della marmitta fragorosa e del tritolo » (p. 79).Si, effectivement, tout s'est d'abord joué dans la langue du fait d'une funeste trahison des clercs, Consolo semble penser que ce n'est qu'avec la langue que l'on peut reconstruire ce que la langue du non-dire politique et la non-langue de la culture de masse ont détruit ${ }^{\text {II }}$.

Et pour qu'on ne puisse pas dire de tous les villages de Sicile, de tous les villages d'Italie, ce que, à la fin des années quatre-vingt, il dit d'Acitrezza qui n'est plus que " morte dell'anima, sigillo d'ogni pianto, arresto del canto, fine del poema, turbinio di parole, suoni privi di senso" (OO, p. 49), il proclame que

Trova solo senso il dire o ridire il male, nel mondo invaso in ogni piega e piaga dal diluvio melmoso e indifferente di parole atone e consunte, con parole antiche o nuove, con diverso accento, di diverso cuore, intelligenza. Dirlo nel greco d'Eschilo, in un volgare vergine come quello di Giacomo o di Cielo o nella lingua pietrosa e aspra d'Acitrezza. (OO, p. 77)

Il ne s'agit pas là d'une simple déclaration de poétique mais d'une véritable déclaration de guerre contre la langue corruptrice du pouvoir, dénoncée précédemment par Pasolini et Sciascia, de la même façon que le refus du récit était une déclaration de guerre contre la pensée unique incarnée par le tout-puissant narrateur, le roman étant le genre littéraire le plus menacé par l'une et par l'autre car, « dès lors qu’il doit nécessairement contenir une valeur communicative, il risque d'être envahi par la communication du pouvoir, il risque d'être entièrement possédé par sa langue » (DQDF, p. 235).

Pour ne pas être possédé par la langue uniformisante de ce pouvoir destructeur de culture et de mémoire dont la disparition des lucioles marque métaphoriquement la naissance, par la langue de la nouvelle société de masse, Consolo entend donc, comme Verga autrefois, inventer un nouveau logos, capable d'aller au-delà de l'idéologie dominante, au-delà de la signification historique et politique, "dans le sens d'une condition humaine

II. À propos de la langue du non-dire inventée par les hommes politiques italiens à l'aube des années quatrevingt, voir la réflexion de Sciascia dans L'affaire Moro, explicitement énoncée p. I5-I6 et développée tout au long de son enquête sur les documents de l'enquête. Quant à la non-langue de la culture de masse ici dénoncée, elle fait l'objet d'une analyse critique plus approfondie de la part de Consolo dans la dernière section de Di qua dal faro intitulée Parole come pietre. 
générale et éternelle. Un langage qui, en allant de la communication vers l'expression, rejoint donc la poésie " $(D Q D F$, p. 229 et p. 282).

De fait, à l'instar de la construction polyphonique qu'il a élaborée en atomisant le récit en une succession horizontale de tranches de vie qui trouvent leur cohérence dans les rapports psychologiques et physiques qui lient les uns aux autres ceux qui les ont vécues et les relient aux lieux où ils ont vécu, Consolo forge une langue multiple en creusant verticalement dans l'épaisseur des stratifications linguistico-culturelles accumulées dans le creuset sicilien. Ainsi, dans le même paragraphe et parfois dans la même phrase, se succèdent des mots d'autrefois et des mots d'aujourd'hui, des mots d'ici et des mots d'ailleurs, dans une infinité de combinaisons qui permet à la fois de capturer au mieux la vraie réalité et d'échapper aux tentatives de récupération par la langue plate et vide du discours dominant, grâce à un "mistilinguisme" que nous avons analysé de façon plus systématique dans d'autres travaux ${ }^{12}$ et dont les citations que nous donnons ici en langue originale donnent un aperçu.

Cependant, cette langue hybride, ce cheval qui recèle dans ses flancs les troupes bigarrées de toutes les langues du bassin méditerranéen à travers les âges et qui est destiné à faire tomber la nouvelle Troie de la kermesse médiatico-littéraire afin qu'Ulysse-Consolo puisse rentrer dans une Ithaque débarrassée des usurpateurs, n'est-elle pas l'un de ses monstres artificiels capables de réveiller les vrais monstres que l'on voit lorsqu'on s'approche de Gela, de vrais monstres à la double nature, issus eux aussi de strates multiples, capables eux aussi de tromper sur leurs origines et leurs fins, et qui ressemblent à s'y méprendre aux Cyclopes et aux Lestrygons qui entravèrent jadis le retour d'Ulysse et annoncèrent naguère le fascisme?

Sono ancora li sparsi i fortini, le casematte della difesa costiera, sembrano, affioranti dalle dune, dai macconi, bianchi di fresca scialbatura, le coperture a calotta, i neri occhi delle feritoie, le teste di giganti, d'arcaici guerrieri che stanno per risorgere o mostri, robot di calcestruzzo, che emergono da ipogei, caserme sotterrranee, avanzano, marciano, distruggono [...] Più avanti, nella vasta landa saudita, sono le teste d'ariete, i lunghi colli delle pompe che vanno su e giù come in un movimento vano e inarrestabile, gli astratti metafisici ingranggi di cui nessuno sa l'origine e il fine. Qui è il teatro dell'abbaglio e dell'inganno, del petrolio favoloso [...] qui il Gela I, Gela 2, Gela 3 [che] accesero Mattei di forza e di

I2. Voir, en particulier, L. Bossi, La voix de la Sicile, entre idiolecte et mistilinguisme (actes du colloque international «Les enjeux du plurilinguisme dans la littérature italienne », CIRILLIS de l'université de Toulouse-Le Mirail, II-I3 mai 2006). Collection de l'ECRIT, CIRILLIS/IL LABORATORIO, Presses de l'université de Toulouse-Le Mirail, 2007. Ead., De Verga à Camilleri : entre sicilitude et sicilianité, les auteurs siciliens font-ils du genre? (actes du Séminaire «Identité(s), langage et modes de pensée », CERCLI de l’université de Saint-Étienne, 7 novembre 2003), dans Identité, langage(s) et modes de pensée, études réunies par Agnès Morini, Publications de l'université de Saint-Étienne, 2004. 
speranza, lo spinsero alla sfida dell'ENI statuale al duro capitalismo dei privati, al Gulf Italia Company, alla Montecatini [...], posero sopra le facce malariche dei contadini $i$ bianchi caschi di plastica operaia.

Da quei pozzi, da quelle ciminiere sopra templi e necropoli, da quei sottosuoli d'ammassi di madrepore e di ossa, di tufi scanalati, cocci dipinti, dall'acropoli sul colle difesa da muraglie, dalla spiaggia aperta a ogni sbarco, dal secco paese povero e obliato parti il terremoto, lo sconvolgimento, parti l'inferno d'oggi (OO, p. 78-79).

Comme l'auraient dit Manzoni, et Sciascia après lui, ainsi allaient les choses en 1994 .

Pour avoir trop bien manipulé la technique de ce sous-produit littéraire de la culture de masse qu'était le roman policier dans les années soixantedix, Sciascia, justement, avait été accusé d'avoir, avec ces préfigurations que sont $\mathrm{Il}$ contesto et Todo modo, provoqué en quelque sorte "l'affaire Moro » alors même que son but était de dénoncer les énigmatiques corrélations dont Moro a été l'acteur et la victime, ainsi que le langage du non-dire qu'il a si bien su utiliser et qui l'a ensuite empêché de se faire comprendre ${ }^{\mathrm{r}}$. Mais depuis, le roman policier et sa structure sont devenus une sorte de schéma narratif unique utilisé non seulement par ceux qui veulent, à la suite de Sciascia, dénoncer les dérives du pouvoir politico-médiatique ${ }^{\mathrm{I} 4}$, mais aussi et surtout par les suppôts de ce même pouvoir dont les ouvrages produits à la chaîne étouffent et excluent toute tentative de subversion et se bornent à entretenir les peurs ataviques et les comportements paranoïaques que le pouvoir a toujours su utiliser à son profit.

De la même façon, alors que Consolo espérait encore, à la fin des années quatre-vingt, pouvoir opposer au déferlement de la communication standardisée, son épos à la structure polyphonique, composée de tout le substrat mythopoétique méditerranéen, et son nouveau logos, sa langue plurielle, faite de toutes les langues d'histoire et de mémoire fondues dans le creuset sicilien, ses tentatives et celles de ceux qui, comme lui, s'efforçaient de faire entendre des voix marginales, ont été noyées dans un multiculturalisme et un communautarisme institutionnels grâce auxquels ces voix ont été récupérées et canalisées.

En démultipliant et en divisant ainsi les enracinements culturels au nom d'une diversité de façade, les serviteurs du pouvoir ont réussi à affaiblir les racines de l'olivier dans lequel Ulysse avait taillé sa couche nuptiale,

13. Voir $A M$, p. I6 et p. 27. Consolo a lui-même fait une analyse des choix narratifs de Sciascia dans la section de Di qua dal faro intitulée Intorno a Leonardo Sciascia, p. 185-208, dans laquelle on lira avec profit les articles Letteratura e potere et Le epigrafi, en particulier p. I99.

I4. Massimo Carlotto ou le collectif Wu Ming, entre autres représentants du genre noir d'inchiesta, exploitent aujourd'hui cette veine et se substituent aux journalistes et aux historiens défaillants. 
qui est aussi le berceau de toute notre civilisation, et, par myopie ou de propos idéologique délibéré, à ne préserver que cette partie du tronc sur laquelle prospère l'oléastre, l'olivier sauvage.

Encore quelques années et la langue de Consolo, dont la complexité sémantique et la richesse lexicale défient déjà la traduction, sera devenue incompréhensible pour la plus grande partie de ses compatriotes; encore quelques années et plus personne ne saura pourquoi Ulysse voulait tant revenir à Ithaque. Et alors, qui dira le mal et dans quelle langue? 